\title{
EFFECT OF POST-ETCHING CLEANING METHODS ON SURFACE MICROMORPHOLOGY AND SHEAR BOND STRENGTH OF COMPOSITE RESIN CEMENT TO FELDSPATHIC CERAMIC BLOCKS
}

\author{
Ahmed Ziada* and Dobbo Csaba**
}

\begin{abstract}
Statement of the problem: Silica-based ceramics, such as feldspathic porcelain are widely introduced as laminate veneers and inlays/onlays due to their excellent esthetic properties. The success in achieving the right bonding protocol is the challenge which contributes to the restorations longevity.
\end{abstract}

Objectives: This study aimed to evaluate the effect of post etching surface cleaning protocol on the surface micromorphology and shear bond strength of resin cement composite to feldspathic ceramic blocks.

Materials and Methods: A total of sixty discs (fifty for the mechanical test and 5 for each scanning microscopic method) of feldspathic ceramic blocks (VITA MARKII) were prepared and etched with $9.5 \%$ hydrofluoric acid for 90 seconds the discs then randomly divided into five groups $(\mathrm{N}=10) \mathbf{H F}$ not subjected to any cleaning step after $\mathrm{HFl}$ etching. The HHU group $37 \%$ phosphoric acid postetched for 30 seconds then immersed in ultrasonic bath for 5 minutes, the HHV group was postetched with $37 \%$ phosphoric acid for 30 seconds then vacuum dried, HV group was vacuum dried only. The HHUV group was postetched with $37 \%$ phosphoric acid for 30 seconds then immersed in ultrasonic bath for 5 minutes then vacuum dried. The surface of each disc was scanned for examination of the surface topography before and after surface treatments. Two different imaging techniques were used: scanning electron microscopy and laser confocal microscopy. Adhesive resin cement (NEXUS 3) was directly bonded to each ceramic disc using a special device providing the same thickness of the cement layer. All the samples were then subjected to shear bond strength tests. Statistical significances between groups were analyzed using one-way ANOVA and (SNK) multiple comparison tests.

Results: The results of the shear bond strength showed that there is significant difference between (HF) group and both HV or HHV groups, while showed no significant differences with

*Lecturer of Fixed Prosthodontics, Fixed Prosthodontic Department, Faculty of Dentistry, Benisuef University, Egypt.

**Proffesor of Radiology and Oral Diagnosis, Semmelweis University, Hungary. 
any other groups where the ultrasonic bath was used. Laser confocal microcopy 3D data analysis showed significant difference in the surface roughness depth between the groups what is subjected to Ultrasonic bath (HHUV and HHU) and the other three groups. the analysis shows significant difference as well between (HF group) and (HV and HHV groups). While was no significant difference between (HV and HHV) groups.

Clinical implication Finding an exact protocol of post etching cleaning is of a big importance nowadays with the increased shifting towards the conservatism and esthetic dentistry and laminate veneers solutions which success depends mainly on the long term bonding strength.

\section{INTRODUCTION}

A big variety of all-ceramic materials and systems are currently available for clinical use. Most of the recent clinical studies concluded superior longterm success of resin-bonded restorations, such as porcelain laminate veneers ${ }^{(1,2)}$, ceramic inlays and onlays. ${ }^{(3,4)}$ feldspathic porcelain are frequently used as laminate veneers and inlays/onlays due to their excellent esthetic properties ${ }^{(3)}$. In spite of its brittleness and limited flexural strength of silica-based ceramics, final adhesive cementation with composite influenced the fracture resistance of the ceramic restoration and the abutment tooth positively. ${ }^{(5,6)}$

A bond strength depends on micromechanical interlocking and chemical bonding to the ceramic surface, roughening and proper cleaning are needed for adequate surface activation ${ }^{(7,8)}$

Using hydrofluoric acid and silane coupling agent together on the surface of the ceramic was claimed to increase the bond strength between the tooth substrate and the ceramic restoration when using resin cement ${ }^{(9,10)}$. However, it has been shown that following hydrofluoric acid removal from the ceramic surface, post etching remnants are formed, which may has an effect on the bond strength ${ }^{(11)}$.

There are studies have introduced different techniques to clean the surface of ceramic, in order to remove the residues left by hydrofluoric acid etching including the use of ultrasonic bath ${ }^{(12,13,14,15,16)}, 37 \%$ phosphoric acid, then rinsing under running water ${ }^{(17,18,19)}$ or $37 \%$ phosphoric acid smear removal and ultrasonic bath respectively ${ }^{(11)}$. There are contradictory results between researches used post-etching protocol ${ }^{(11,12,13,14,15,16,17,18,19)}$.

Therefore this study aimed to evaluate: a) the shear bond strength between the feldspathic ceramic and the adhesive system after pretreatment of the ceramic surface with different steps of the postetching, b) the surface changes between groups by scanning electron microscopy (SEM) and Laser confocal microscopy (LCM).

\section{MATERIAL AND METHODS}

\section{Sample preparation}

Cutting: Sixty discs were cut from feldspathic cad blocks Vita MarkII (VITA Zahnfabrik H. Rauter GmbH \& Co. KG, Spitalgasse, SäckingenGermany) of $2 \mathrm{~mm}$ thickness were prepared using the automated precise saw (BUEHLER, Illinois, US). The ingot was held by special holder while the saw was automated to cut with the desired thickness by adjusting the digital meter. The same standard load was used for all the samples while sawing.

Polishing: In order to achieve the surface roughness of $1 \mu \mathrm{m}$, the surface of each sample went through three stages of polishing:

Stage 1: each surface was subjected to low-speed polishing continuously for $5 \mathrm{~min}$ with a grinding and polishing beta machine (BUEHLER- MIT, Illinois, US). Silicon carbide papers (BUEHLER, Illinoi, USA) of 3 sizes (grit 1200, 1000 and 600) were used from coarse to fine for polishing each surface; 
Stage 2: each surface was polished using the same machine but with a suede polishing disc wetted with polycrystalline diamond polishing suspension (BUEHLER metdi, Illinoi, USA) continuously for 5 min to achieve the surface roughnessof $3 \mu \mathrm{m}$.

Stage 3: the same polishing method as stage 2 was applied except that the polycrystalline diamond polishing suspension (BUEHLER metdi, Illinoi, USA) of $1 \mu \mathrm{m}$ was used to achieve the surface roughness of $1 \mu \mathrm{m}$.

Surface treatment and cleaning methods: All the sixty samples were etched with $9.5 \%$ hydrofluoric acid for 90 seconds, the discs then randomly divided into five separated groups $(\mathrm{N}=10)$. HFl group not subjected to any cleaning step after etching. HHU group: $37 \%$ phosphoric acid for 30 seconds then immersion in ultrasonic bath for 5 minutes. HHV group: $37 \%$ phosphoric acid for 30 seconds then vacuum drying. HV group: vacuum dried only. HHUV group: $37 \%$ phosphoric acid for 30 seconds then immersion in ultrasonic bath for 5 minutes then vacuum drying. All the groups what subjected to vacuum had the same vacuum drying protocol, for 24 hours in vacuumed container while the samples was inside a glass gar. All the samples were put inside the vacuumed container for drying in the same time all together and removed together to standardize the time and condition of dryness.

\section{SURFACE ANALYSIS}

Surfaces of the discs were scanned with two methods after surface treatment:

Scanning electron microscopy (SEM): The discs were gold sputter coated in a sputter coater (BALTEC SCD. 005, England) before examination under a SEM (Joel Scanning Machine, ST 20, JOEL LTD.; Tokyo, Japan). The treated surfaces were examined at $\mathrm{x} 500$ and x1500 magnification and the images were captured using Semaphore computer software Digital slow scan image recording system, version 4.01 (LEAD technologies, Jeol Sundbyberg, Sweden).
Laser Confocal microscopy (LCM): The surfaces were examined with LCM after applying a 1.25 $\mathrm{mM}$ Maleimide fluorescens dye under low vacuum condition (Alex fluor 488 c5 dye material, Thermo Fisher scientific, Waltham, MA.USA) and left the dye to dry on the surface. For the surface image scan illumination at $488 \mathrm{~nm}$ wavelength was used. Region of interests (ROIs) with the same size were taken from five different areas of sample surface. The 3D image sets of dye (five ROIs per sample) transformed to DICOM and fed into the CTAn software (Bruker, Kontik, Belgium) for micromorphological analysis the mean of five samples was used to define the percent dye volume.

\section{Cementation of the luting cement}

A commercial silane coupling agent (Kerr corporation, West Collins, Orange, CA, USA) was applied to the treated surface of each ceramic disc, allowed to set for 1 min before drying with oil-free air jet.

NX3 adhesive resin cement base (Kerr corporation, West Collins, Orange, CA, USA) supplied in a syringe was mixed with the catalyst in the spiral mixing tip and applied using a plastic instrument onto the surface of each ceramic disc which also contained the coupling agent. The cement was pressed against the disc before a standardized load $(20 \mathrm{kN})$ was applied using a specially designed loading device. The device made of two horizontal shelves attached through two rigid vertical metal columns. The upper shelf has a hole in the middle through which a metal cylinder can pass in upand down direction vertically free way. This cylinder has a lower flat base that rests on the disc sample and a upper disc-shaped end that act as seat holds the $20 \mathrm{kN}$ load during the cementation procedure.

The resin cement was left to set without disturbance for $1.5 \mathrm{~min}$ before the excess removal in its gel form. The resin cement was light-cured at room temperature for $10 \mathrm{~s}$ at $45^{\circ}$ from all directions 
under the load and was subsequently allowed to set for another $10 \mathrm{~s}$ after the load was removed. This way the total setting time of $20 \mathrm{~s}$ was guaranteed according to the manufacturers' instructions.

\section{Shear bond strength test}

For the bond strength test specially designed rectangular terminal shear test was designed samples were placed one by one on a computer controlled testing machine (Model LRX-plus; Lloyd Instruments Ltd., Fareham, UK) with a 5 kN applied load. Results were listed using computer software (Nexygen-MT; Lloyd Instruments). Each Sample was secured to the lower non movable part by securing screw. Shearing test was performed compressively by applying load at ceramic -ceramic interface with a metallic mono beveled chisel rod fixed to the superior movable part of the testing machine at cross-head speed $.5 \mathrm{~mm} / \mathrm{m}$. needed load for debonding was listed in Newton.

\section{Shear bond strength calculation:}

The point of failure was divided by bonding area to represent the bond strength in $\mathrm{MPa}: \tau=\mathrm{P} /$ A where; $\tau=$ shear bond strength (MPa, $\mathrm{P}=$ load at failure $(\mathrm{N}) \mathrm{A}=$ width $\mathrm{x}$ breadth $\left(\mathrm{mm}^{2}\right)$
Statistics: A one-way ANOVA test was performed followed by a Tukey test to show significant difference between the five tested groups.

\section{RESULTS}

The results of the shear bond strength as shown in Figure 1. Results showed significant difference between HF group and both HV or HHV groups, while showed no significant differences with other groups where the ultrasonic bath was used.

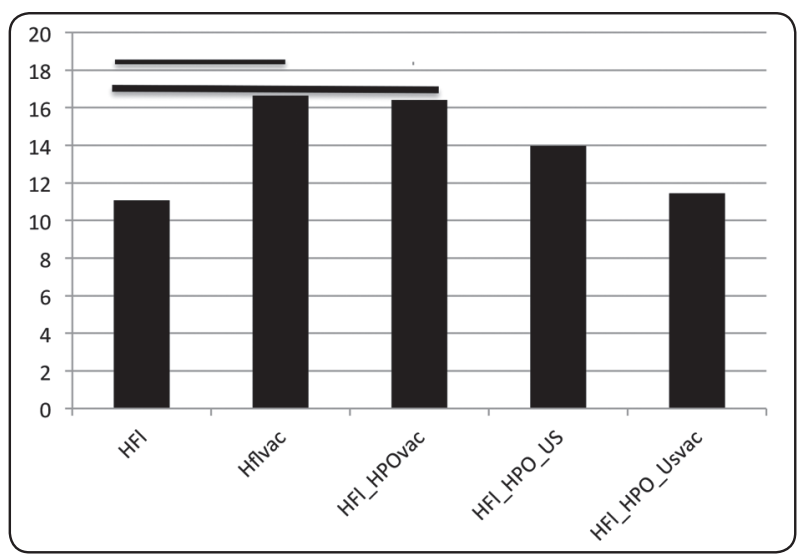

Fig. (1) Shear bond strength measurements. Results showed significant differences between $\mathrm{HF}$ group and both HV or HHV groups, while showed no significant differences with any other groups where the ultrasonic bath was used. $(\mathrm{p}<0.05)$
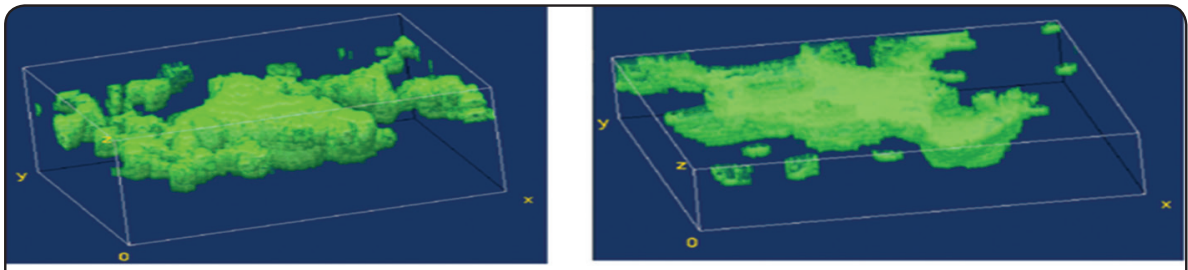

HF

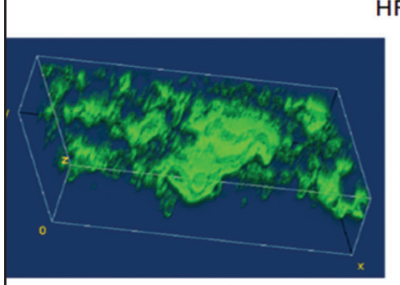

HHV
HV

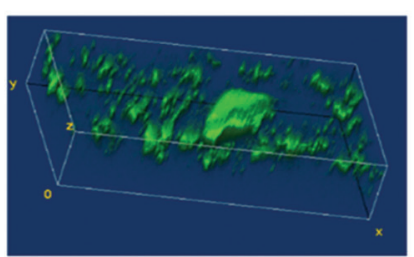

HHUV

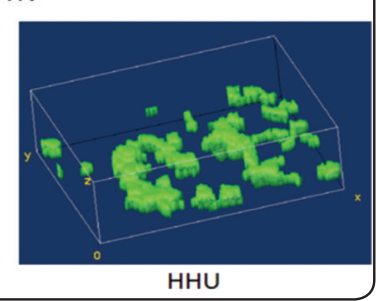

Fig. (2) Laser confocal microscopy 3D view of dye dispersion Green dye extended in the acid etched surface cavities under low vacuum condition. The extent and thickness of dye are definitely reduced in ultrasonic groups (HHUV and HHU) 


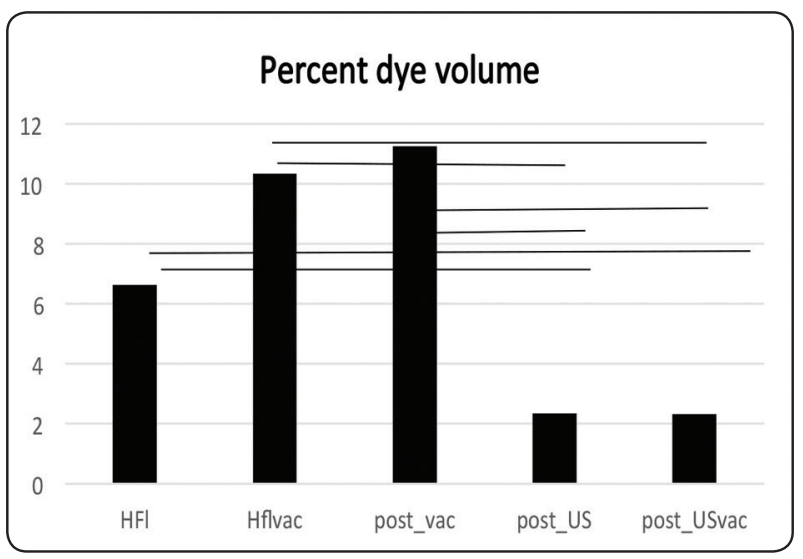

Fig. (3) Laser confocal microscopy 3D data anyalysis expressed in percent dye volume showed significant difference in the surface cavity area and depth between the groups what is subjected to Ultrasonic bath (HHUV and HHU) and the other three groups. There is significant difference between HFL group and HV or HHV groups. $(\mathrm{n}=5) \quad(\mathrm{p}<0.05)$
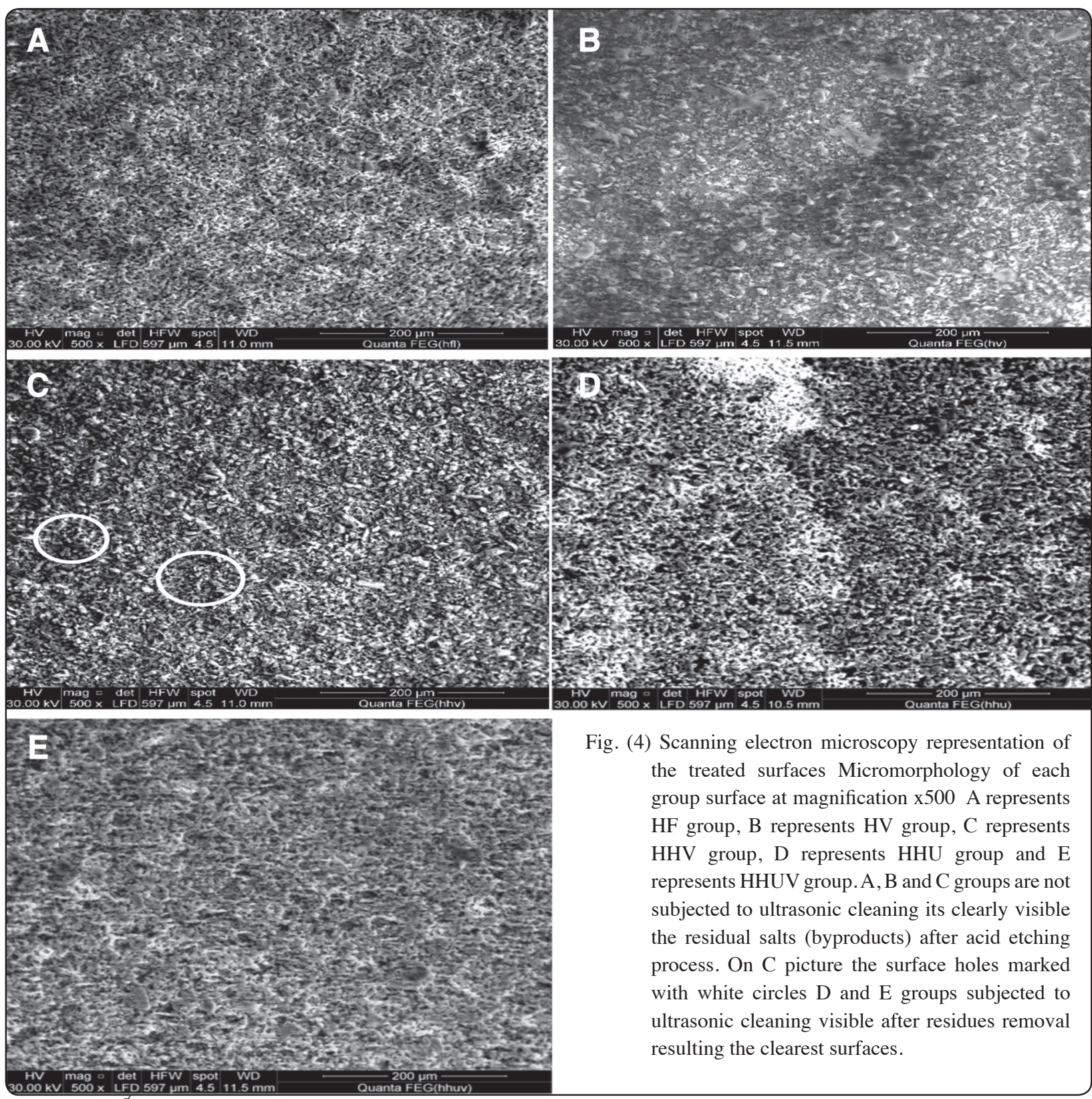

Fig. (4) Scanning electron microscopy representation of the treated surfaces Micromorphology of each group surface at magnification $\mathrm{x} 500$ A represents HF group, B represents HV group, C represents HHV group, D represents HHU group and $\mathrm{E}$ represents HHUV group. A, B and C groups are not subjected to ultrasonic cleaning its clearly visible the residual salts (byproducts) after acid etching process. On $\mathrm{C}$ picture the surface holes marked with white circles D and E groups subjected to ultrasonic cleaning visible after residues removal resulting the clearest surfaces. 


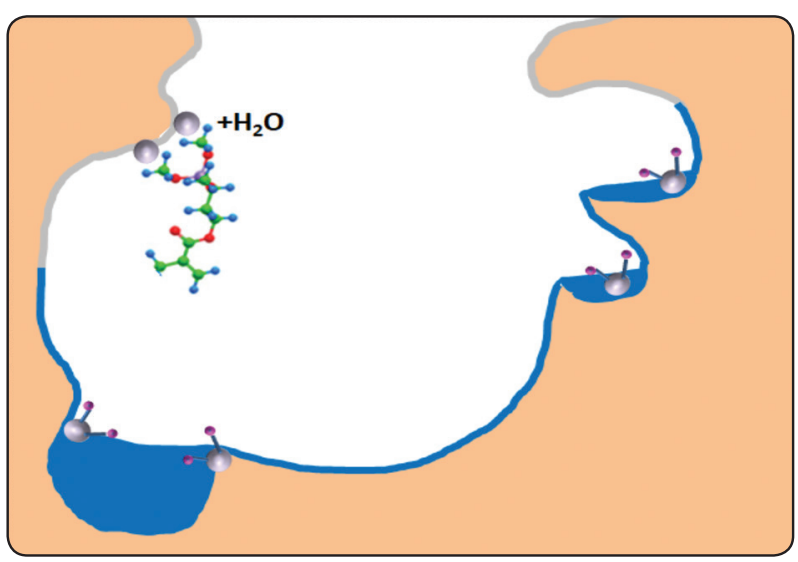

Fig. (5) Schematic representation of inactivation of silan on wet surfaces of the etched and/or postetched cavities Blue represents wet surfaces, grey represents dry surfaces where the chemical condensation reaction goes off.

\section{DISCUSSION}

A bond strength depends on micromechanical interlocking and chemical bonding to the ceramic surface, roughening and proper cleaning are needed for adequate surface activation ${ }^{(7,8)}$

Resin cements and internal ceramic surface treatment are increase bonding between ceramic and tooth structure ${ }^{(20,21)}$. This treatment change the smooth surface and the low surface energy to increase the flow of the bonding agents to make adequate chemical or mechanical retention ${ }^{(22)}$. Dental ceramics microstructure are made up of loosened crystals or granules ${ }^{(2)}$. Feldspathic ceramic containing of feldspath $(65 \%)$, quartz $( \pm 25 \%)$ and metal oxides $( \pm 10 \%)$. The response to acid etching is possible due to the high content of the glass matrix which allow the creation of micro porosities and surface irregularity that improve adhesion ${ }^{(17)}$. Hydrofluoric acid treatment promotes ceramic surface area forming uneven contour and raise the surface energy for micromechanical interlocking of the resin material ${ }^{(11,23)}$. Hydrofluoric acid etching istargeting only the silicate particles in the glass matrix that's why it's broadly used with fieldspathic glass ceramics producing hexaflourosilicates thus creating a porous surface to enhance the micromechanical bonding with resin cement
${ }^{(17,19,23,24)}$. surface etching with hydrofluoric acid at a concentration of $9 \%$ to $10 \%{ }^{(12,25,26,27,28)}$ and etching time ranged between one ${ }^{(9,12,14,19,29)}$ and two minutes was the recommended protocol for pretreatment of feldspathic ceramics ${ }^{(14,19)}$. In the present study $9 \%$ of hydrofluoric acid was applied for one minute. Nevertheless researchers studying etching protocol stated non significant influence between the times used considering etching time not less than one minute , ${ }^{(14,19)}$.

Following etching of silica-based ceramics, it has been dimonstrated that hydrofluoric acid leads to the formation of insoluble byproducts, such as silica and fluoride salts which may remain on the ceramic surface ${ }^{(9)}$. These remnants may interfere with the bond strength between the ceramic and the substrate onto which it is cemented ${ }^{(9,30,31)}$. This could explain the remaining residues that appeared on SEM images with our groups HF, HV and HHV that subjected to hydrofluoric acid etch and vacuum dryness without ultrasonic baths. In order to remove byproducts following hydrofluoric acid etching, some authors used ultrasonic washing ${ }^{(12-16)}$ $37 \%$ phosphoric acid smear ${ }^{(32)}$, rinsing with running water $^{(17-19)}$ or a combination of $37 \%$ phosphoric acid smear and ultrasonic washing ${ }^{(11)}$. Our observation supports this statement since phosphoric acid treatment alone (our HHV group) couldn't remove byproducts effectively without ultrasonic washing from the etched surface (Fig 4C). Phark and coworkers ${ }^{(15)}$ reported that shear bond strength is not affected by byproducts or cleaning procedures, which is in agreement with our result in this study that showed no significant difference in the shear bond strength between both HHU, HHUV groups and $\mathrm{HF}$ group.

The present study demonstrated no significant difference regarding the cleaning methods of the dental ceramic surface, however, the significant difference was found when using vacuum drying methods. Groups subjected to vacuum dryness e.g. HV, HHV had significant difference in bond strength when compared HF group. The SEM shows clearing effect of the ultrasonic bath in removing 
the debris from the surface since our results show an agreement with Caballero and co-workers ${ }^{(33)}$ who concluded that micro-shear bond strength of feldspathic ceramics and adhesive resin cement not influenced. Ultrasonic cleaning and the residual debris remaining on the surface had no influence on the micro-shear bond strengths ${ }^{(33)}$.

In this study the vacuum groups increased the microshear strength rather due to complete dryness than the ultrasonic cleaning resulted in clean surface but more flat even surface by removing the most superficial layer of treated surface which may be influenced the final shear bong strength. This even surface developed by ultrasonic cleaning observed in this study is in line with Caballero ${ }^{(33)}$ and Giraldo ${ }^{(34)}$ report that on their samples when ultrasonic bath where applied the surfaces became flattened ${ }^{(33,34)}$. In the present study the laser confocal microscope data analysis explained this previously mentioned statement as it shows that HHUV and HHU groups had the most flattened surface and attracted the least fluorescent dye material hence the ultrasonic procedure had reduced the volume of cavities. The remaining water film takes its place on the surface which compromises the bonding of the silane later on. However, the completely dried surface has not this side effect of ultrasonic bath which was proved with the groups HV and HHV as both had significantly higher volume of cavity on surface than the other groups subjected to ultrasonic bath resulting the significantly higher values with the group HF in the shear test.

\section{CONCLUSION}

Within the limitation of this study we could conclude that, regardless the post acid etching cleaning protocol used, the complete dryness under vacuum if possible has effect on the shear bond strength of feldspathic ceramics.

The remaining residues (the reaction byproducts between acid etch and ceramic surface) does not affect the shearbond strength for feldspathic ceramics.

\section{REFERENCES}

1. Cho GC, Donovan TE, Chee WW. Clinical experiences with bonded porcelain laminate veneers. J Calif Dent Assoc 1998;26:121-7.

2. Peumans M, Van Meerbeek B, Lambrechts P, Vanherle G. Porcelain veneers: a review of the literature. J Dent 2000;28:163-77.

3. Blatz MB. Long-term clinical success of all-ceramic posterior restorations.Quintessence Int 2002;33:415-26.

4. Sjogren G, Molin M, van Dijken JW. A 5-year clinical evaluation of ceramic inlays (Cerec) cemented with a dual-cured or chemically cured resin composite luting agent. Acta Odontol Scand 1998;56:263-7.

5. el-Mowafy $\mathrm{O}$. The use of resin cements in restorative dentistry to overcome retention problems. J Can Dent Assoc 2001;67:97-102.

6. Jensen ME, Sheth JJ, Tolliver D. Etched-porcelain resinbonded fullveneer crowns: in vitro fracture resistance. Compendium 1989;10:336-8,340-1, 344-7.

7. Semmelmann JO, Kulp PR. Silane bonding porcelain teeth to acrylic.J Am Dent Assoc 1968;76:69-73.

8. Chen JH, Matsumura H, Atsuta M. Effect of etchant, etching period, and silane priming on bond strength to porcelain of composite resin. OperDent 1998;23:250-7.

9. Fabianelli A, Pollington S, Papacchini F, Goracci C, Cantoro A, Ferrari M, et al. The effect of different surface treatments on bond strength between leucite reinforced feldspathic ceramic and composite resin. J Dent. 2010; 38:39-43.

10. PollingtonS, Fabianelli A,Noort RV, Microtensile bond strength of a resin cement to a novel flourcanasite glassceramic following different surface treatment. Dent Mater,2010;26:864-72.

11. Magne P, Cascione D. Influence of post -etching cleaning and connecting porcelain on the microtensile bond strength of composite resin to feldspathic porcelain. J Prosthet Dent.2006;96:354-61

12. Brentel AS, Özcan M, Valandro LF, Alarça LG, Amaral R, Bottino MA. Microtensile bond strength of a resin cement to feldspathic ceramic after different etching and silanization regimens in dry and aged conditions. Dent Mater. 2007;23:1323-31.

13. Dundar M,Ozcan M, Gokce B, Comlekoglu E. comparison of two bond strength testing methodologies for bilayered all-ceramics. Dental Mater. 2007;23:630-6. 
14. Menezes FC, Borges GA, Valentino TA, Oliveira MA, Turssi CP,Correr-Sobrinho L. Effect of surface treatment and storage on the bond strength of different ceramic systems. Braz J Oral Sci. 2009;8:119-23.

15. Phark JH, Duarte S Jr,Kahn H, Blatz MB, Sadan A. Influence of contamination and cleaning on bond strength to modified zirconia. Dent Mater.2009;25:1541-50.

16. Yadav S, Upadhyay M, Borges GA,Roberts WE. Influece of ceramic (feldspathic) surface teatments on the micro-shear bond strength of composite resin.Angle Orthod .2010;80:765-70.

17. Kukiattrakoon B, Thammasitboon K. Optimal acidulated phosphate fluoride gel etching time for surface treatment of feldspathic porcelain: on shear bond strength to resin composite. Eur J Dent. 2012;6:63-9.

18. Melo RM, Valandro L, Bottino MA. Microtensile bond strength of a repair composite to leucite-reinforced feldspathic ceramic. Braz Dent J. 2007;18:314-9.

19. Trakyali G, Malkondu O,Arun T. Effect of different silanes and acid concentrations on bond strength of brackets to porcelain surfaces. Eur J Orthod. 2009;31:402-6.

20. Elzohairy AA,Saber MH, Abdalla AI, Felizer AJ. Efficacy of microtensile versus microshear bond testing for evaluation of bond strength of dental adhesive systems to enamel. Dent Mater.2010;26:848-54.

21. Frankenberger R, Reinelt C, Petschelt A, Krämer N. Operator vs.material influence on clinical outcome of bonded ceramic inlays.Dent Mater.2009;25:960-8.

22. Queiroz JR, Benetti P, Özcan M, Oliveira LF, Della Bona A, Takahashi FE, et al. Surface characterization of feldspathic ceramic using ATR FT-IR and ellipsometry after various silanization protocols. Dent Mater. 2012;28:189-96.

23. YA dav S,Upadhayay M,Borges GA,Roberts WE. Influence of ceramic (feldspathic) surface treatments on the micro-shear bond strength of composite resin. Angle Orthod. 2010;80:765-70.

24. Dalkiz M, Sipahi C, Beydemir B. Effects of six surface treatment methods on the surface roughness of a low-fusing and an ultra-low-fusing feldspathic ceramic material. J Prosthodont. 2009; 18:217-22.

25. Attia A,Lehmann F,Kern M. Influence of surface conditioning and cleaning methods on resin bonding to zirconia ceramic. Dent Mater. 2011;27:207-13.

26. Ersu B, Yuzugullu B, Yazici AR, Canay S. Surface roughness and bond strength of glass- infiltrated aluminaceramics prepared using various surface treatments. J Dent. 2009;37:848-56.

27. Hooshmand T, Parvizi S, Keshvad A. Effect of surface acid etching on the biaxial flexural strength of two hot-pressed glass ceramics. J Prosthodont. 2008;17:415-9.

28. Saraç S, Elekdaq-Turk S, Saraç D, Turk T. Surface conditioning methods and polishing techniques effect on surface roughness of a feldspar ceramic. Angle Orthod. 2007;77:723-8.

29. Yi Y, Kelly JR. Failure responses of a dental porcelain having three surface treatments under three stressing conditions. Dent.Mater. 2011;27:1252-8.

30. Monticelli F, Toledano M, Osorio R, Ferrari M. Effect of temperature on the silane coupling agents when bonding core resin to quartz fiber posts. Dent Mater.2006;22:1024-8.

31. Shimada Y, Yamaguchi S, Tagami J. Micro-shear bond strength of dual-cured resin cement to glass ceramics. Dent Mater.2002;18:380-8.

32. Quaas AC, Yang B, Kern M. Panavia F 2.0 bonding to contaminated zirconia ceramic after different cleaning procedures. Dent Mater. 2007;23:506-12.

33. Caballero HS, Cecilia PT, Fabiana MG, Flavia LB. Micro-shear bond strength and surface micromorphology of a feldspathic ceramic treated with different cleaning methods after hydrofluoric acid etching. JAppl Oral Sci.2014;22(2):85-90

34. Giraldo TC,Vanessa RV, Mauricio PC. Active and passive application of phosphoric acid on the bond strength of Lithium Disilicate. Brazilian Dental Journal. 2016;27(1):90-94. 